PROCEEDINGS OF THE

AMERICAN MATHEMATICAL SOCIETY

Volume 130, Number 8, Pages 2219-2227

S 0002-9939(02)06514-0

Article electronically published on February 7, 2002

\title{
SUBNORMAL SUBGROUPS OF THE GROUPS OF RATIONAL POINTS OF REDUCTIVE ALGEBRAIC GROUPS
}

\author{
GOPAL PRASAD AND ANDREI S. RAPINCHUK \\ (Communicated by Rebecca Herb)
}

\begin{abstract}
We prove that for a reductive algebraic group $G$ over an infinite field $K$, the group of rational points $G(K)$ does not contain any noncentral finitely generated normal subgroups.
\end{abstract}

\section{INTRODUCTION}

We recall that a subgroup $\mathcal{N}$ of an abstract group $\mathcal{G}$ is said to be subnormal if there exists a descending chain of subgroups (called a subnormal series)

$$
\mathcal{G}=\mathcal{G}_{0} \supset \mathcal{G}_{1} \supset \cdots \supset \mathcal{G}_{s}=\mathcal{N}
$$

such that $\mathcal{G}_{i+1} \triangleleft \mathcal{G}_{i}$ for all $i=0,1, \ldots, s-1$. Papers [1]-2] were devoted to various particular cases of the question of whether the group $\mathcal{G}=G L_{n}(D)$, where $D$ is a division algebra and $n \geqslant 1$, can have finitely generated noncentral subnormal subgroups. Finally, it was proven in [10 that if $D$ is finite dimensional over its center, then any finitely generated subnormal subgroup of $G L_{n}(D)$ must be central. The goal of this note is to show that the latter result is a particular case of a general statement about finitely generated subnormal subgroups in the group of rational points of reductive algebraic groups (and not only over fields, but also over semilocal rings arising from valuations) 1

Before stating our main theorem, we would like to state some conventions and notations to be used throughout the paper. All valuations considered will be realvalued (in other words, will have height one). Given a nonarchimedean valuation $v$ of a field $K$, we let $\mathcal{O}_{v}$ denote the corresponding valuation ring. We tacitly assume that every linear algebraic $K$-group $G$ comes with a certain fixed $K$-embedding $G \subset \mathbf{G L}_{n}$ which will allow us to consider freely the group $G(A):=G \cap G L_{n}(A)$ of $A$-points for any subring $A \subset K$. It should be noted that although the group $G(A)$ may depend on the choice of an embedding $G \subset \mathbf{G L}_{n}$, all our results remain true for any embedding.

Received by the editors March 5, 2001.

2000 Mathematics Subject Classification. Primary 20G15, 20G30, 22E46.

${ }^{1}$ Unfortunately, extending results about normal subgroups from the group $D^{*}$, where $D$ is a finite dimensional divison algebra, to the groups of points of arbitrary connected reductive algebraic groups is not always this simple. More specifically, it was shown in [16] that any finite quotient of $D^{*}$ is solvable; whether or not this fact generalizes to the group $G(K)$, where $G$ is an arbitrary connected reductive group over an infinite field $K$, is an interesting and difficult open problem. 
Theorem 1. Let $G$ be a connected reductive algebraic group defined over an infinite field $K, S$ be a finite (possibly, empty) set of nontrivial inequivalent nonarchimedean valuations of $K$, and $\mathcal{O}=\bigcap_{v \in S} \mathcal{O}_{v}$. If $N$ is a subnormal subgroup of $G(\mathcal{O})$, not contained in the center $Z(G)$, then $N$ cannot be contained in a finitely generated subgroup of $G(K)$.

When $S=\emptyset$, then by definition $\mathcal{O}=K$, so we immediately obtain the following.

Corollary 1. Let $G, K$ be as in Theorem 1 . Then no noncentral subnormal subgroup of $G(K)$ is contained in a finitely generated subgroup of $G(K)$.

Applying this corollary to the group $G=\mathbf{G L}_{n, D}$ associated with a finite dimensional division algebra $D$, we recover the result of [10].

The proof of Theorem 1 is based on the possibility to embed any finitely generated integral domain into either the ring of $p$-adic integers (in characteristic zero) or the ring of formal power series in one variable over a finite field (in positive characteristic); see Proposition 11 The first application of this method (known to us) is due to Platonov [12] who established with its help the nonsurjectivity (on the groups of rational points) of an isogeny between two algebraic groups over finitely generated fields. Later it was also used for different purposes (cf., for example, [15], $\S 4)$. Proposition 1 is proven in $\S 3$ by a suitable adaptation of Platonov's argument. The first-named author would like to thank Adrian Wadsworth and Brian Conrad who supplied him with two different proofs of this proposition. Richard Pink has pointed out that results from Appendix B of his paper [11] can be used to give yet another proof.

The two authors proved the above theorem independently. As the ideas used by them were similar, they decided to write this note jointly. Both the authors were partially supported by the NSF and BSF (Israel-USA). The first author proved the theorem while he visited the Forschungsinstitut für Mathematik, ETH, Zurich. He would like to thank this institute for its hospitality and support. We are also grateful to the referee for his suggestions that helped to improve the exposition.

\section{ON WEAK APPROXIMATION IN ALGEBRAIC GROUPS}

The proof of Theorem 1 uses some information about weak approximation in reductive algebraic groups which we will recall now. Let $G$ be a connected reductive algebraic group defined over an infinite field $K$. Given a $K$-variety $X$ and a finite set $S$ of (nontrivial) inequivalent valuations of $K$, we let

$$
X(S):=\prod_{v \in S} X\left(K_{v}\right)
$$

where $K_{v}$ is the completion of $K$ with respect to $v$, endowed with the direct product topology. The closure of the diagonally embedded $X(K)$ in $X(S)$ will be denoted by $\overline{X(K)}^{(S)}$.

It was shown by Harder 8 that $\overline{G(K)}^{(S)}$ contains $\prod_{v \in S} N_{v}$ for some open normal subgroups $N_{v} \subset G\left(K_{v}\right)$. Availing this opportunity, we would like to point out another feature of $\overline{G(K)}^{(S)}$ here (we note, however, that the proof of Theorem 1 does not actually require the full strength of this statement).

Theorem 2. There exists an integer $m>0$ ( $m$ depending only on the absolute rank of $G$ ) such that for any finite set $S=\left\{v_{1}, \ldots, v_{d}\right\}$ of inequivalent valuations 
of $K$, the closure $\overline{G(K)}^{(S)}$ contains $G(S)^{(m)}$, the subgroup generated by the $m$-th powers $x^{m}, x \in G(S)$.

We need the following.

Lemma 1. Let $T$ be a $K$-torus with the splitting field $L$, and $l=[L: K]$. Given a finite set $S$ of inequivalent valuations of $K$, the closure $\overline{T(K)}^{(S)}$ contains $T(S)^{l}$.

Proof. The idea of this proof goes back to Serre and Harder (see Hauptlemma in [8]). We give the argument here for the sake of completeness (cf. the proof of Lemma 9.3 in our paper [14]). We let $N=R_{L / K}(T)$, and consider the exact sequence

$$
1 \rightarrow M \longrightarrow N \stackrel{\eta}{\longrightarrow} T \rightarrow 1,
$$

in which $\eta$ is the "norm" map (cf., for example, the proof of Proposition 6.7 in 13]); note that $M:=\operatorname{Ker} \eta$ is a $K$-torus. Corresponding to (1), we have the following commutative diagram with exact rows:

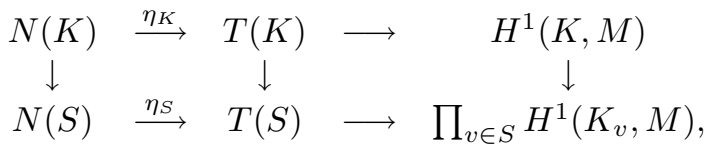

where for an extension $\mathcal{K} / K, H^{1}(\mathcal{K}, M)$ denotes the first Galois cohomology

$$
H^{1}\left(\operatorname{Gal}\left(\mathcal{K}_{s} / \mathcal{K}\right), M\left(\mathcal{K}_{s}\right)\right)
$$

$\mathcal{K}_{s}$ being the separable closure of $\mathcal{K}$. Since $T$ is $L$-isomorphic to $\left(\mathbf{G L}_{1}\right)^{r}, r=\operatorname{dim} T$, we have a $K$-isomorphism

$$
N \simeq R_{L / K}\left(\mathbf{G L}_{1}\right)^{r} .
$$

We need the following two consequences of (3). First, $N$, and therefore also $M$, splits over $L$. Hence, by Hilbert's Theorem 90,

$$
H^{1}(\mathcal{K}, M)=H^{1}(L \mathcal{K} / \mathcal{K}, M),
$$

so the exponent of $H^{1}(\mathcal{K}, M)$ divides $l=[L: K]$, for any extension $\mathcal{K} / K$. In particular, the exponent of $\prod_{v \in S} H^{1}\left(K_{v}, M\right)$ divides $l$. This, in view of (2), implies that $T(S)^{l} \subseteq \eta_{S}(N(S))$. Secondly, (3) implies that $N$ is a smooth $K$-rational variety, hence it has the weak approximation property with respect to any finite set of inequivalent valuations (cf. Proposition 7.3 in [13]), i.e., $\overline{N(K)}^{(S)}=N(S)$. Thus,

$$
T(S)^{l} \subseteq \eta_{S}(N(S)) \subseteq{\overline{\eta_{K}(N(K))}}^{(S)} \subseteq \overline{T(K)}^{(S)},
$$

as claimed.

Proof of Theorem Q As in the proof of Lemma 9.3 of [14], we observe that there exists an integer $m>0$ such that for any maximal $K$-torus $T$ of $G$, the degree $[L: K]$ of its minimal splitting field $L$ divides $m$. Indeed, the (finite) Galois group $\operatorname{Gal}(L / K)$ injects into Aut $X(T)$, where $X(T)$ is the character group of $T$. Since Aut $X(T) \simeq G L_{r}(\mathbb{Z})$, where $r=\operatorname{dim} T$ is the rank of $G$, the order of $\operatorname{Gal}(L / K)$ will divide, for example, $m=\left[G L_{r}(\mathbb{Z}): G L_{r}(\mathbb{Z}, 3)\right]$ as the congruence subgroup $G L_{r}(\mathbb{Z}, 3)$ is torsion-free by Minkowski's Lemma (see, for example, Lemma 4.19 in [13]). We will show that this number $m$ will work.

Let $R=\prod_{v \in S} R(v)$, where $R(v) \subset G\left(K_{v}\right)$ is the set of elements $x_{v} \in G\left(K_{v}\right)$ such that there exists a maximal $K_{v}$-torus $T_{v}$ of $G$ for which $x_{v} \in T\left(K_{v}\right)^{m}$. We 
claim that $R \subset \overline{G(K)}^{(S)}$. Take any $x \in R, x=\left(x_{v}^{m}\right)$, where $x_{v} \in T_{v}\left(K_{v}\right)$ for some maximal $K_{v}$-torus $T_{v}$ of $G$. We fix a neighborhood $W$ of $x$ and pick a neighborhood of the identity $\Omega=\prod_{v \in S} \Omega_{v}$ in $G(S)$ such that $\left\{\omega^{-1} x \omega \mid \omega \in \Omega\right\} \subset W$. Let $\mathcal{T}$ be the variety of maximal tori of $G$. We observe that for any valuation $v$ of $K$ and any torus $T \in \mathcal{T}\left(K_{v}\right)$, the map $\phi: G\left(K_{v}\right) \rightarrow \mathcal{T}\left(K_{v}\right)$ given by: $g \stackrel{\phi}{\mapsto} g \cdot T$, where $g \cdot T=g^{-1} T g$, is open in the $v$-adic topology. Indeed, since $\mathcal{T}$ is a quotient of $G$, the differential $d_{g} \phi: T_{g}(G) \rightarrow T_{\phi(g)}(\mathcal{T})$ is surjective for any $g \in G$, so the required openness follows from the implicit (or inverse) function theorem (see, for example, [17], p. 85). Thus, for any $v \in S$, the set $\Omega_{v} \cdot T_{v}$ is open in $\mathcal{T}\left(K_{v}\right)$. On the other hand, since $\mathcal{T}$ is rational over $K$ (a theorem due to Grothendieck, cf. [5], Theorem 7.9, and also [6] for characteristic zero case) and smooth, it has the weak approximation property (Proposition 7.3 in 13 ), and therefore there exists a torus

$$
T \in \mathcal{T}(K) \cap \prod_{v \in S}\left(\Omega_{v} \cdot T_{v}\right) .
$$

Then there exists $\omega=\left(\omega_{v}\right) \in \Omega$ such that $T=\omega_{v}^{-1} T_{v} \omega_{v}$ for each $v \in S$, implying that $\omega^{-1} x \omega \in T(S)^{m}$. In view of Lemma 1 and our choice of $m$, we have that $\omega^{-1} x \omega \in \overline{T(K)}(S)$, and therefore $W$, being a neighborhood of $\omega^{-1} x \omega$, meets $T(K)$, hence also $G(K)$. This proves the claim.

Now, we will show that $\bar{R}^{(S)}$ contains the set $G(S)^{m}$ of the $m$-th powers of elements of $G(S)$. For this, it suffices to show that the closure $\overline{R(v)}^{(v)}$ of $R(v)$ in $G\left(K_{v}\right)$ contains $G\left(K_{v}\right)^{m}$. It is well-known that the subset $U$ of $G$ of regular semi-simple elements is a Zariski-open $K$-subvariety (this is obvious from the fact that an element $g \in G$ is regular semi-simple if, and only if, the multiplicity of the root 1 of the characteristic polynomial of $\operatorname{Ad} g$ is minimal possible, i.e. it is equal to the rank of $G$ ). Then by Lemma 3.2 of [13], $U\left(K_{v}\right)$ is dense in $G\left(K_{v}\right)$, and therefore $\left(U\left(K_{v}\right)\right)^{m}$ is dense in $G\left(K_{v}\right)^{m}$, for any $v \in S$. However, if $x=y^{m}$ with $y \in U\left(K_{v}\right)$, then $T=C_{G}(y)^{\circ}$, the identity component of the centralizer of $y$, is a maximal $K_{v}$-torus and $y \in T\left(K_{v}\right)$ (cf. [4, 11.12), and the required fact follows.

Now as $\overline{G(K)}(S)$ is a subgroup containing $G(S)^{m}$, it contains the subgroup $G(S)^{(m)}$ generated by the latter. This completes the proof of Theorem 2

Corollary 2. Let $V$ be a finite set of (nontrivial) nonarchimedean valuations of $K, \Gamma=G\left(\mathcal{O}_{V}\right)$, where $\mathcal{O}_{V}=\bigcap_{v \in V} \mathcal{O}_{v}$. For any nontrivial valuation $v_{0} \notin V$, the closure $\bar{\Gamma}^{\left(v_{0}\right)}$ of $\Gamma$ in $G\left(K_{v_{0}}\right)$ contains $G\left(K_{v_{0}}\right)^{(m)}$, where $m$ is as in Theorem 2 .

Indeed, let $S=V \cup\left\{v_{0}\right\}$ and

$$
U=\prod_{v \in V} G\left(\widetilde{\mathcal{O}_{v}}\right) \times G\left(K_{v_{0}}\right)
$$

where $\widetilde{\mathcal{O}_{v}}$ is the valuation ring of $K_{v}$. Since $U$ is an open and closed subgroup of $G(S)$, we conclude that

$$
\overline{G(K)}^{(S)} \cap U={\overline{G(K) \cap U^{(S)}}}^{(S)} \bar{\Gamma}^{(S)} .
$$

It follows now from Theorem 2 that $\bar{\Gamma}^{(S)}$ contains $\{1\} \times \cdots \times\{1\} \times G\left(K_{v_{0}}\right)^{(m)}$, so the closure of $\Gamma$ in $G\left(K_{v_{0}}\right)$ contains $G\left(K_{v_{0}}\right)^{(m)}$. 


\section{Embedding Finitely GENERATED IntEgRAL DOMAins INTO COMPACT RINGS}

Proposition 1. Let $R$ be a finitely generated infinite integral domain, $K$ be its field of fractions, and $L / K$ be a finite separable extension. Then there exists a discrete valuation $v$ of $K$ such that:

1) the completion $K_{v}$ is locally compact;

2) $R \subset \tilde{\mathcal{O}}_{v}$, the valuation ring of $v$ in $K_{v}$;

3) $L_{w}=K_{v}$ for any extension $w \mid v$.

Proof. Let $K_{0}$ (resp., $R_{0}$ ) be the prime subfield of $K$ (resp., the prime subring of $R$ ). Since $K_{0}$ is perfect, there exists a transcendence basis $s_{1}, \ldots, s_{l}$, where $l \geqslant 0$, of $K$ over $K_{0}$ such that $K$ is separable over $P:=K_{0}\left(s_{1}, \ldots, s_{l}\right)$ (cf. [9], Ch. X, $\S 6)$. Then $L / P$ is also separable; in particular, $L=P[\alpha]$ for some $\alpha \in L$. Let $A:=R_{0}\left[s_{1}, \ldots, s_{l}\right]$ and $B:=A[\alpha]$. Since $R$ is finitely generated, there exists $a \in A$ such that

$$
R \subset B\left[\frac{1}{a}\right]
$$

By adjusting $a$, we can assume in addition that $f(x)$, the minimal polynomial of $\alpha$ over $P$, has all the coefficients in $A\left[\frac{1}{a}\right]$. The polynomial $f(x)$ is prime to its derivative $f^{\prime}(x)$, so there exist polynomials $p(x), q(x) \in A[x]$ such that

$$
p(x) f(x)+q(x) f^{\prime}(x)=b
$$

for some nonzero $b \in A$. Set $c=a b$.

Let $C$ denote $\mathbb{Z}$ if $K$ is of characteristic zero, and $K_{0}[t]$, where $t$ is an indeterminate, if $K$ is of positive characteristic. Pick $c_{1}, \ldots, c_{l} \in C$, not all in $K_{0}$ if this field is of positive characteristic, such that for the homomorphism $\varepsilon: A \rightarrow C$, defined by $s_{i} \mapsto c_{i}$, one has $\varepsilon(c) \neq 0$. Then $F:=$ the field of fractions of $\varepsilon(A)$, is a global field. We now observe that given a finite separable extension $\mathcal{F} / F$, there exist infinitely many valuations $u$ of $F$ such that for any extension $w$ of $u$ to $\mathcal{F}$, the completions $\mathcal{F}_{w}$ and $F_{u}$ coincide. (This can be easily seen by applying the Tchebotarev's Density Theorem (cf. [7], Ch. 5) to the minimal Galois extension of $F$ containing $\mathcal{F}$.) We also recall that given an element $r \in F^{*}$, for all but finitely many nonarchimedean valuations $u$ of $F$, one has $u(r) \geqslant 0$ (cf. [3], Ch. II, Lemma 12.1), i.e. $r$ belongs to the valuation ring of $u$. Using the first of these facts for the splitting field $\mathcal{F}$ (over $F$ ) of the polynomial $g(x)=f^{\varepsilon}(x)$ obtained by applying the natural extension of $\varepsilon$ to the coefficients of $f(x)$, in combination with the second fact, one can find a valuation $u$ of $F$ such that $\varepsilon\left(c_{1}\right), \ldots, \varepsilon\left(c_{l}\right)$ lie in the valuation ring of $u, u(c)=0$, and $g(x)$ splits over the (locally compact) completion $F_{u}$ into linear factors. Let $\widetilde{\mathcal{O}_{u}}$ be the valuation ring of $u$ in $F_{u}, \mathfrak{P}_{u}$ be its maximal ideal, and $k_{u}=\widetilde{\mathcal{O}_{u}} / \mathfrak{P}_{u}$ be the (finite) residue field. We observe that all the roots of $g(x)$ belong, in fact, to $\widetilde{\mathcal{O}_{u}}$ (because $g(x)$ is monic with coefficients in $\widetilde{\mathcal{O}_{u}}$, and $\widetilde{\mathcal{O}_{u}}$ is integrally closed), implying that the residue polynomial $\bar{g}(x)$ is a product of linear factors over $k_{u}$.

Since $\widetilde{\mathcal{O}_{u}}$ is uncountable, one can pick $t_{1}, \ldots, t_{l} \in \widetilde{\mathcal{O}_{u}}$ to be algebraically independent over $K_{0}$ and satisfy $t_{i} \equiv c_{i}\left(\bmod \mathfrak{P}_{u}\right)$ for all $i=1, \ldots, l$. Let $\sigma: P \rightarrow F_{u}$ be the embedding which sends $s_{i}$ to $t_{i}$. We claim that the polynomial $h(x):=f^{\sigma}(x)$ splits over $F_{v}$ into linear factors. Indeed, by our construction $\bar{h}(x)=\bar{g}(x)$. On the 
other hand, it follows from (2) that

$$
\overline{p^{\sigma}}(x) \bar{h}(x)+\overline{q^{\sigma}}(x) \bar{h}^{\prime}(x)=\bar{b} \neq \overline{0},
$$

which implies that $\bar{h}(x)$ is prime to its derivative $\bar{h}^{\prime}(x)$. It follows now from Hensel's Lemma that $h$ splits over $\widetilde{\mathcal{O}_{u}}$ into linear factors. Then, for any embedding $\bar{\sigma}$ of $L$ into the algebraic closure $\overline{F_{u}}$ extending $\sigma$, one has $\bar{\sigma}(L) \subset F_{u}$. Now, if $v$ is the valuation of $K$ obtained by pulling back $u$ under one of these embeddings, then $K_{v}=F_{u}$ and $\widetilde{\mathcal{O}_{v}}=\widetilde{\mathcal{O}_{u}}$; moreover, $L_{w}=F_{u}=K_{v}$ for any extension $w \mid v$. Since $\bar{\sigma}(\alpha)$ is a root of $h(x)$, we have $\bar{\sigma}(\alpha) \in \widetilde{\mathcal{O}_{v}}$, and then (1) implies that $R \subset \widetilde{\mathcal{O}_{v}}$ as $v(a)=0$. The proof of the proposition is complete.

Remark. In his paper 12, Platonov established that any finitely generated field can be embedded into a locally compact field. Proposition 1 gives a slightly more refined result, i.e. that a finitely generated integral domain can be embedded into the valuation ring of such a field. This was achieved by minor modifications of his argument.

\section{Proof of Theorem 1}

We begin with the following two lemmas.

Lemma 2. Let $G$ be a connected $K$-simple $K$-group. If a subgroup $F \subset G(K)$ is Zariski-dense in $G$, then any subnormal subgroup $N$ of $F$, not contained in $Z(G)$, is also Zariski-dense in $G$.

Indeed, let

$$
F=N_{0} \supset N_{1} \supset \cdots N_{s-1} \supset N_{s}=N
$$

be a subnormal series. Taking the closure (denoted ${ }^{`}$ ) in the Zariski $K$-topology, we obtain the following subnormal series

$$
G=\check{F}=\check{N}_{0} \supset \check{N}_{1} \supset \cdots \supset \check{N}_{s-1} \supset \check{N}_{s}=\check{N} .
$$

However, each of the $\check{N}_{i}$ 's is defined over $K$ ([4], AG.14.4), so since $G$ is $K$-simple, we obtain by induction

$$
G=\check{N}_{0}=\ldots=\check{N}_{s}=\check{N}
$$

as claimed.

Lemma 3. Let $K$ be an infinite field, $S$ be a finite set of nontrivial inequivalent valuations of $K$, and $\mathcal{O}=\bigcap_{v \in S} \mathcal{O}_{v}$. For any connected reductive $K$-group $G$, the group $G(\mathcal{O})$ is Zariski-dense in $G$.

Proof. This statement is well-known for $S=\emptyset$, i.e. for $\mathcal{O}=K$; cf. 4], Corollary 18.3. The general case is obtained by minor modifications and uses the same idea, viz. that the variety $G$ is unirational over $K$ which means that there exists a dominant $K$-rational map $f: \mathbb{A}^{l} \rightarrow G$ of the affine space $\mathbb{A}^{l}$ of an appropriate dimension (4], Theorem 18.2). Without any loss of generality we may assume that $f(0, \ldots, 0)=e$ (the identity). Clearly, $f$ induces a continuous map $f_{S}: U(S) \rightarrow$ $G(S)$, where $U \subset \mathbb{A}^{l}$ is a Zariski $K$-open subset (the domain of $f$ ) containing $(0, \ldots, 0)$. Since $G(\mathcal{O})$ is open in $G(K)$ with respect to the topology induced from $G(S)$, there exists $r \geqslant 0$ such that for

$$
\mathfrak{a}:=\{x \in K \mid v(x)>r \quad \forall v \in S\} \cup\{0\},
$$


one has $f(\mathfrak{a}, \ldots, \mathfrak{a}) \subset G(\mathcal{O})$. But since the valuations in $S$ are nontrivial and inequivalent, it easily follows from the weak approximation theorem (cf. [9], Ch.XII, $\S 1)$ that $\mathfrak{a}$ is infinite. This implies that $\mathfrak{a} \times \cdots \times \mathfrak{a}$ is Zariski-dense in $\mathbb{A}^{l}$, and since $f$ is dominant, we obtain the Zariski density of $G(\mathcal{O})$ in $G$.

Proof of Theorem 1 . We let $N \subset G(\mathcal{O})$ denote a subnormal subgroup, not contained in $Z(G)$, where $G, K$, and $\mathcal{O}$ are as in the statement of the theorem. Thus, there exist a subnormal series

$$
G(\mathcal{O})=N_{0} \supset N_{1} \supset \cdots \supset N_{s-1} \supset N_{s}=N,
$$

where $N_{i+1} \triangleleft N_{i}$ for $i=0, \ldots, s-1$. From now on, we assume in addition that $N$ is contained in some finitely generated subgroup $\Gamma$ of $G(K)$. Then there exists a finitely generated subring $R$ of $K$ such that: 1) $\Gamma \subset G(R)$, and 2) $G$ is defined over the field of fractions $K^{\prime}$ of $R$. To ensure the first condition, it is sufficient to pick a finite system of generators $x_{1}, \ldots, x_{r}$ of $\Gamma$ and include in $R$ the entries of the matrices $x_{i}^{ \pm 1}, 1 \leqslant i \leqslant r$. For the second condition, we observe that by Hilbert's Basis Theorem, the ideal $\mathfrak{a} \subset K\left[x_{11}, \ldots, x_{n n},\left(\operatorname{det}\left(x_{i j}\right)\right)^{-1}\right]$ of $K$-regular functions on $\mathbf{G L}_{n}$ vanishing on $G$ is generated by a finite system of polynomials, and it is sufficient to include in $R$ the coefficients of all these polynomials. It is now clear that there exists a finitely generated subring $R$ satisfying both conditions. Replacing $K$ with $K^{\prime}, \mathcal{O}$ with $\mathcal{O}^{\prime}=\mathcal{O} \cap K^{\prime}$, and observing that $\mathcal{O}^{\prime}=\bigcap_{v \in S^{\prime}} \mathcal{O}_{v}^{\prime}$, where $S^{\prime}$ is the finite set of valuations of $K^{\prime}$ obtained by first restricting the valuations in $S$ to $K^{\prime}$ and then dropping all trivial valuations and picking one representative from each class of equivalent ones, and $\mathcal{O}_{v}^{\prime}$ is the valuation ring for $v \in S^{\prime}$, we may assume that $K$ is the field of fractions of its finitely generated subring $R$ such that $N \subset G(R)$.

Pick a maximal $K$-torus $T$ of $G$, and let $L$ denote its minimal splitting field. By Proposition 1, one can find a valuation $v_{0}$ of $K$ such that $R$ is contained in the valuation ring $\widetilde{\mathcal{O}_{v_{0}}}$ of $v_{0}$ in $K_{v_{0}}$ and $L \subset K_{v_{0}}$. Then $N \subset G\left(\widetilde{\mathcal{O}_{v_{0}}}\right)$, and $T$ splits over $K_{v_{0}}$. As $N \not \subset Z(G)$, one can find a $K$-morphism $\pi: G \rightarrow H$ onto a $K$-simple adjoint group $H$ such that $\pi(N) \not \subset Z(H)$. Then it follows from Lemma 3 that $\pi(G(\mathcal{O}))$ is Zariski-dense in $H$, so by Lemma 2, $N^{\prime}:=\pi(N)$ is also dense in $H$. Consider the subnormal series obtained by applying $\pi$ to (1):

$$
\pi(G(\mathcal{O}))=N_{0}^{\prime} \supset N_{1}^{\prime} \supset \cdots \supset N_{s-1}^{\prime} \supset N_{s}^{\prime}=N^{\prime},
$$

where $N_{i}^{\prime}:=\pi\left(N_{i}\right), 1 \leqslant i \leqslant s$. To complete the proof, we need one lemma. Prior to stating the lemma we recall that given a field $\mathcal{K}$ with a real-valued valuation $v$, a subset $X \subset \mathbf{M}_{n}(\mathcal{K})$ is said to be bounded if there exists $r \in \mathbb{R}$ such that for every matrix $\left(a_{i j}\right) \in X$, one has $v\left(a_{i j}\right) \geqslant r$ for all $i, j \in\{1, \ldots, n\}$.

Lemma 4. Let $H$ be a semi-simple adjoint algebraic group over a field $\mathcal{K}$ endowed with a valuation $v$. If a Zariski-dense subgroup $\Delta \subset H(\mathcal{K})$ is bounded (in terms of some matrix $\mathcal{K}$-realization of $H), 2$ then so is its normalizer $\mathcal{N}:=N_{H(\mathcal{K})}(\Delta)$.

Proof. We fix a matrix $\mathcal{K}$-realization $H \hookrightarrow \mathbf{G L}_{n}$, and let $A$ denote the subalgebra of the matrix algebra $\mathbf{M}_{n}$ spanned by $H$. The adjoint action of $H$ on $A$ gives rise to a morphism of algebraic groups $\phi: H \rightarrow \mathbf{G L}(A)$ which is, in fact, an isomorphism onto the image as $H$ is adjoint. Since $\Delta$ is Zariski-dense in $H$, it also spans $A$.

\footnotetext{
${ }^{2}$ As isomorphisms between algebraic groups are given by polynomial functions, this notion of boundedness, in fact, does not depend on the choice of a matrix realization.
} 
We pick a basis $\left\{e_{1}, \ldots, e_{d}\right\}$, where $d=\operatorname{dim} A$, of $A$ contained in $\Delta$, and use it to identify $\mathbf{G L}(A)$ with $\mathbf{G L}_{d}$ in the sequel. Then $\phi$ is defined over $\mathcal{K}$.

Now, let $\mathcal{O}$ be the valuation ring of $v$, and let $\Lambda$ be the $\mathcal{O}$-submodule of $\mathbf{M}_{n}(\mathcal{K})$ generated by $\Delta$. Since $\Delta$ is bounded, then so is $\Lambda$ (the "bound" that works for $\Delta$ will also work for $\Lambda$ ). This implies that there exists $s \in \mathbb{R}$ such that for any $a \in \Lambda$, the coefficients in the expansion $a=\sum_{i=1}^{d} \alpha_{i} e_{i}$ satisfy $v\left(\alpha_{i}\right) \geqslant s$ for all $i=1, \ldots, d$. (Indeed, the expansions of the elements of $\Lambda$ have bounded coefficients with respect to the standard basis of $\mathbf{M}_{n}(\mathcal{K})$, so the same remains true for any other basis of $\mathbf{M}_{n}(\mathcal{K})$; on the other hand, the set $\left\{e_{1}, \ldots, e_{d}\right\}$ can be enlarged to a basis of $\mathbf{M}_{n}(\mathcal{K})$, but the expansion of the elements of $\Lambda$ will not involve other elements of the basis.) For $x \in \mathcal{N}$, as $\phi(x)$ stabilizes $\Lambda$, the entries of the matrix $\phi(x):=\left(x_{i j}\right)$ satisfy $v\left(x_{i j}\right) \geqslant s$. Hence, $\phi(\mathcal{N})$ is bounded. Since $\phi$ is an isomorphism, $\mathcal{N}$ itself is bounded.

Returning to the series (2), we have $N^{\prime} \subset \pi\left(G\left(\tilde{\mathcal{O}}_{v_{0}}\right)\right)$, so $N^{\prime}$ is a bounded subgroup of $H\left(K_{v_{0}}\right)$ with respect to the valuation $v_{0}$ of $\mathcal{K}:=K_{v_{0}}$. Since $N^{\prime}$ is also Zariski-dense, we obtain from Lemma 4 that $N_{s-1}^{\prime}$ is bounded. Continuing by induction, we obtain that all the terms of (2) are bounded; in particular, $\pi(G(\mathcal{O}))$ is bounded, and therefore its closure $\overline{\pi(G(\mathcal{O}))}^{\left(v_{0}\right)}$ in $H\left(K_{v_{0}}\right)$ is also bounded. On the other hand, let $T^{\prime}=\pi(T)$, and let $t$ be the order of the torsion subgroup of the quotient $X(T) / \pi^{*}\left(X\left(T^{\prime}\right)\right)$ of the character group $X(T)$ of $T$. Then $\pi\left(T\left(K_{v_{0}}\right)\right) \supset T^{\prime}\left(K_{v_{0}}\right)^{t}$. At the same time, according to Corollary 2, we have $T\left(K_{v_{0}}\right)^{m} \subset \overline{G(\mathcal{O})}^{\left(v_{0}\right)}$ for some integer $m>0$. These two facts together imply that

$$
T^{\prime}\left(K_{v_{0}}\right)^{m t} \subset \overline{\pi(G(\mathcal{O}))}^{\left(v_{0}\right)}
$$

and therefore $\overline{\pi(G(\mathcal{O}))}^{\left(v_{0}\right)}$ is not bounded as $T$, and hence also $T^{\prime}$, splits over $K_{v_{0}}$. A contradiction, which proves the theorem.

\section{REFERENCES}

1. S. Akbari and M. Mahdavi-Hezavehi, Normal subgroups of $G L_{n}(D)$ are not finitely generated, Proc. AMS, 128(1999), 1627-1632. MR 2000j:16052

2. S. Akbari, M. Mahdavi-Hezavehi and M.G. Mahmudi, Maximal subgroups of $G L_{1}(D)$, J. Algebra, 217(1999), 422-433. MR 2000d:16025

3. Algebraic Number Theory, Proc. of an instructional conference organized by the London Mathematical Society, ed. by J.W.S. Cassels and A. Frölich, Academic Press, 1967. MR 35:6500

4. A. Borel, Linear Algebraic Groups, GTM 126, Springer-Verlag, 1991. MR 92d:20001

5. A. Borel and T.A. Springer, Rationality properties of linear algebraic groups II, Tohoku Math. J., 20(1968), 443-497. MR 39:5576

6. C. Chevalley, On algebraic group varieties, J. Math. Soc. Japan, 6(1954), 303-324. MR 16:672g

7. M.D. Fried and M. Jarden, Field Arithmetic, Springer-Verlag, Heidelberg, 1986. MR 89b:12010

8. G. Harder, Eine Bemerkung zum schwachen Approximationssatz, Archive Math., 19(1968), 465-471. MR 39:2767

9. S. Lang, Algebra, Addison-Wesley, 1965. MR 33:5416

10. M. Mahdavi-Hezavehi, M.G. Mahmudi and S. Yasamin, Finitely generated subnormal subgroups of $G L_{n}(D)$ are central, J. Algebra, 225 (2000), 517-521. MR 2000j:20097

11. R. Pink, The Mumford-Tate conjecture for Drinfeld-modules, Publ. Res. Inst. Math. Sci., 33(1997), 393-425. MR 98f:11062

12. V.P. Platonov, Dieudonné's conjecture and the nonsurjectivity on k-points of coverings of algebraic groups, Soviet Math. Dokl., 216 (1974), 986-989. MR 50:7368 
13. V.P. Platonov and A.S. Rapinchuk, Algebraic Groups and Number Theory, Pure and Applied Math. N 139, Academic Press, 1993. MR 95b:11039

14. G. Prasad and A.S. Rapinchuk, Computation of the metaplectic kernel, Publ. Math. IHES, 84(1996), 90-187. MR 98i:22026

15. A.S. Rapinchuk, Combinatorial theory of arithmetic groups, Preprint 20(420), 1990, Institute of Mathematics of the Academy of Sciences of BSSR (Minsk).

16. A.S. Rapinchuk, Y. Segev and G.M. Seitz, Finite quotients of the multiplicative group of a finite dimensional division algebra are solvable, preprint.

17. J-P. Serre, Lie algebras and Lie groups, Lect. Notes Math., 1500, Springer-Verlag, 1992. MR 93h:17001

Department of Mathematics, University of Michigan, Ann Arbor, Michigan 48109

E-mail address: gprasad@math.Isa.umich.edu

Department of Mathematics, University of Virginia, Charlottesville, Virginia 22904

E-mail address: asr3x@weyl.math.virginia.edu 